\title{
Independent effects of weight gain and fetal programming on metabolic complications in adults born small for gestational age
}

\author{
T. Meas $\cdot$ S. Deghmoun $\cdot$ C. Alberti $\cdot$ E. Carreira $~$ \\ P. Armoogum • D. Chevenne $\cdot$ C. Lévy-Marchal
}

Received: 4 November 2009/Accepted: 8 December 2009/Published online: 29 January 2010

(C) Springer-Verlag 2010

\begin{abstract}
Aims/hypothesis Insulin resistance (IR) and the metabolic syndrome (MS) have been reported in adults as a consequence of being born small for gestational age (SGA). The process seems to be initiated early in life; however, little is known about the progression of MS and IR in young adults. We hypothesised that being born SGA would promote a greater progression over time of IR and MS, reflecting not only the gain in weight and fat mass but also the extension of the fetal programming process.

Methods Participants were selected from a community-based cohort and born full-term either SGA (birthweight $<10$ th percentile) or appropriate for gestational age $(25$ th $<$ birthweight $<75$ th percentile). A total of 1,308 individuals were prospectively followed between the ages of 22 and 30 years.

Results At both ages, individuals born SGA were more insulin-resistant and showed a significantly higher prevalence of MS. Over the 8 year follow-up, the risk of developing MS was twofold higher in those SGA, after
\end{abstract}

T. Meas $(\bowtie) \cdot$ S. Deghmoun • E. Carreira • C. Lévy-Marchal

INSERM Unité U690, Hôpital Robert Debré,

48 Boulevard Sérurier,

75019 Paris, France

e-mail: taly.meas@lrb.aphp.fr

T. Meas $\cdot$ C. Alberti $\cdot$ C. Lévy-Marchal

Université Paris,

Paris, France

C. Alberti $\cdot$ P. Armoogum

Unité d'Epidémiologie Clinique, AP-HP, Hôpital Robert Debré,

Paris, France

D. Chevenne

Service de Biochimie Hormonale, AP-HP, Hôpital Robert Debré, Paris, France adjustment for gain in BMI, whereas the progression of IR was not significantly affected by the birth status.

Conclusions/interpretation Our data suggest that metabolic disorders in SGA individuals are amplified by the weight gain with time when adults, both probably resulting from fetal programming. Moreover, the modest increase in IR contrasts with the constant and much higher prevalence of MS.

Keywords Fetal programming - Insulin resistance · Metabolic syndrome $\cdot$ SGA
Abbreviations
AGA Appropriate for gestational age
BW Birthweight
DI Disposition index
IR Insulin resistance
MS Metabolic syndrome
SGA Small for gestational age

\section{Introduction}

Being born small for gestational age (SGA) has been associated with an increased risk of insulin resistance (IR) and metabolic syndrome (MS) [1, 2]. MS is a clustering of hypertension, dyslipidaemia, elevated BP and abdominal obesity. Since the first observation by Barker et al. [3], several investigators have reported the association between low birthweight (BW) and an increased incidence of components of MS, either during childhood [4] or when adults $[5,6]$. Data from our group show that people born SGA are more insulin-resistant and have a sixfold increased rate of MS at the age of 22 years compared with pairs born appropriate for gestational age (AGA) [7]. 
These metabolic complications are seen as the consequences of a specific sequence of poor fetal growth leading to reduced size at birth followed by early postnatal catch-up. The mismatch between a poor intrauterine environment and more favourable postnatal conditions could result in abnormal body composition, which in turn will promote IR. Individuals born SGA suffer from a severe reduction of fat mass at birth $[8,9]$, but a large proportion of them will experience a rapid postnatal catch-up in growth during the first year of life $[10,11]$. The association between metabolic disorders and low BW appears stronger when small size at birth is followed by early catch-up growth [7, 12, 13].

The role of gain in weight beyond the phase of catch-up growth needs also to be considered. In many cross-sectional studies in adults, the strongest association between BW and the metabolic outcome is found when a statistical adjustment for adult size is incorporated [14, 15]. In children, an interaction between BW and subsequent gain in BMI or fat mass during childhood has been described for IR, systolic BP and serum cholesterol [16, 17]. Similar data were reported in a cohort of 20-year-old South Africans [18]. This argues that low BW per se is not the only factor implicated, but other factors such as the change in size between birth and adulthood could also play a key role. Moreover, several data have shown an association between low BW and modification of later body composition in young adults born SGA [19, 20]. Recently, we have reported that being born SGA affects body composition when adults [21]. Individuals born SGA show an accelerated gain in BMI and waist circumference, which in turn results in higher body fat content with a central distribution at the age of 30 years. Altogether, these results suggest that being born SGA has metabolic consequences evolving beyond the period of catch-up. If this is the case, the metabolic complications would progress more rapidly in adults when resulting from SGA. Although many cross-sectional studies found an association between BW and MS, little is known about the progression of the metabolic disorders in adults. In the Dutch famine cohort, individuals were studied at 50 and 57 years old [22]. The authors found that individuals with low BW had a greater age-related progression of glucose intolerance, and a large part of this decline could be attributed to an increase in BMI.

In order to determine the respective impact of fetal growth and weight gain in young adults we have prospectively followed the progression over time of MS and IR between ages 22 and 30 years in a cohort of individuals born either SGA or AGA. We hypothesised that the metabolic complications would progress faster in the individuals born SGA, resulting from the interaction between fat accumulation with age and the metabolic complications of the small size at birth, both having fetal origins.

\section{Methods}

Participants Individuals were identified from a communitybased cohort of young adults aimed at investigating the longterm consequences of being born SGA. Briefly, individuals were derived from a population-based registry of the metropolitan area of the city of Haguenau in France [7]. This registry included information about all pregnancies and deliveries occurring in the maternity unit of the city hospital from 1971 to 1985 . The SGA group includes 734 singleton individuals born between 32 and 42 weeks of gestation with $\mathrm{BW}<10$ th percentile for sex and gestational age according to the local growth standard curves. The AGA group is made of singleton individuals born between 32 and 42 weeks of gestation, with BW between the 25th and the 75th percentiles and who were the first babies in the registry born immediately after an individual born SGA. The AGA group included 886 individuals.

The initial observation took place at a mean age of 22 years, the results of which have already been published [7]. The second observation was conducted between April 2005 and December 2008. Among 1,620 eligible individuals for the prospective follow-up, 54 had moved to an unknown address, 160 refused further participation, 90 were not eligible (concomitant disease, pregnancy, living abroad, chronic diseases or chronic treatment inducing metabolic disorders, pre-existing diabetes such as type 1 diabetes or secondary to cystic fibrosis). A total of 1,308 (593 SGA and 715 AGA) agreed to participate in the second visit (rate of participation $80.7 \%$; Fig. 1). All eligible individuals included in the cohort were contacted by phone or by mail. When individuals declined to participate, their current weight was

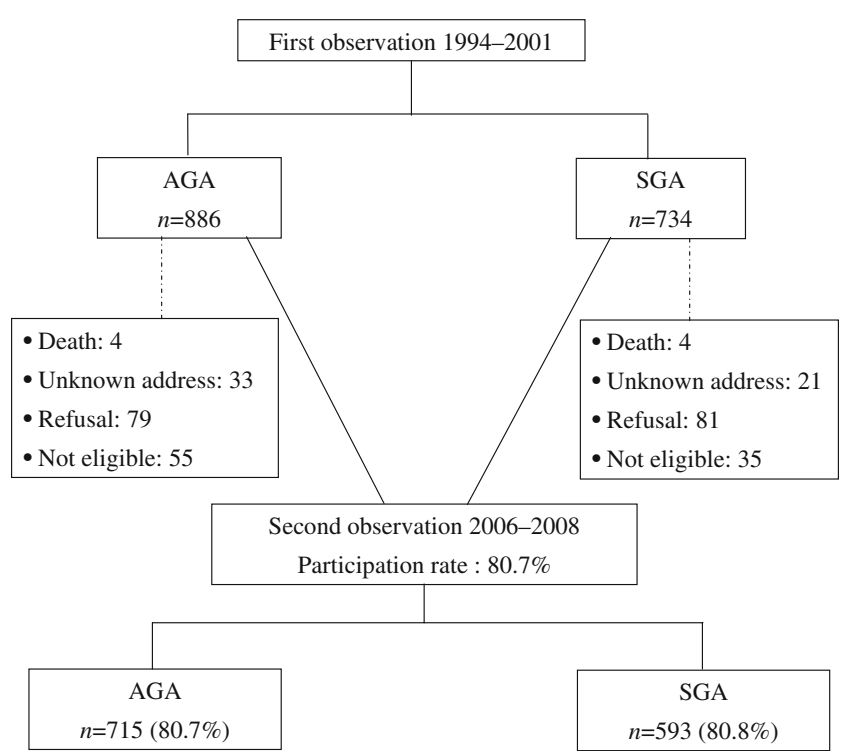

Fig. 1 Flow chart of the follow-up of the SGA and AGA participants between ages 22 and 30 years 
recorded. We have compared the 1,308 individuals who participated in the second visit with the 312 who did not. The proportion of AGA and SGA was comparable. The proportion of women was slightly increased in the nonparticipants $(53.6 \%$ vs $46 \%)$. Birth data and metabolic variables were similar in the two groups. Self-reported data, during a phone call, were obtained in 142 non-participant individuals at the time of the second observation. BMI was also comparable in the non-participant SGA or AGA individuals $\left(24.9 \pm 4.9\right.$ vs $\left.23.9 \pm 4.3[\mathrm{SD}] \mathrm{kg} / \mathrm{m}^{2}\right)$ or participants SGA/AGA $\left(24.2 \pm 5.2\right.$ vs $\left.24.0 \pm 4.33 \mathrm{~kg} / \mathrm{m}^{2}\right)$.

Clinical characteristics of the study population at birth were previously described in details [7]. The individuals were all born full-term and sex distribution did not differ between the AGA and SGA groups. According to the selection criteria, individuals born SGA were lighter $(2,627 \pm$ 296 vs $3,366 \pm 274 \mathrm{~g})$, shorter $(47.7 \pm 2$ vs $50.3 \pm 1 \mathrm{~cm})$ and thinner $\left(11.6 \pm 1.1 \mathrm{vs} 13.3 \pm 0.8 \mathrm{~kg} / \mathrm{m}^{2}\right)$ at birth than individuals born AGA.

Study design Participants attended the two medical visits at the municipal hospital of the city of Haguenau. For both visits, the same three trained nurses recorded information about medical history using a standardised questionnaire. Body weight was measured with a portable scale and height with a wall-mounted stadiometer. Weight for height was assessed as BMI $\left(\mathrm{kg} / \mathrm{m}^{2}\right)$. Waist circumference was measured at the level of the umbilicus and hip circumference at the level of the greater trochanter.

Percentage of body fat mass was measured using a multifrequency bioelectrical impedance meter. All measurements were carried out with participants fasting, lying in a supine position on a flat, non-conductive bed by using tetrapolar technique (Quadscan 4000; Bodystat). Two electrodes were placed on the right wrist one just proximal to the third metacarpophalangeal joints (positive) and one the wrist next to the ulnar head (negative). Two electrodes were placed on the right ankle with one just proximal to the third metatarsophalangeal joint (positive) and one between medial and lateral malleoli (negative). Multifrequency (5, 50,100 and $200 \mathrm{kHz}$ ) currents were introduced from the positive leads and travelled throughout the body to the negative leads. The percentage of body fat was calculated using the manufacturer's software. The CVs for percentage body fat measured at $12 \%$ and $35 \%$ were $2.3 \%$ and $0.6 \%$ for within-individual precision and $3.5 \%$ and $1.1 \%$ for inter-individual precision, respectively.

BP was measured in the right arm of seated individuals after a 30 min rest, using an automated device (Dinamap; Critikon, Neuilly-Plaisance, France) and a cuff of recommended size for the mid-upper arm circumference. Three measurements were made at $1 \mathrm{~min}$ intervals and the average of the last two measurements was used in the analysis.
Blood samples were collected after an overnight fast for measurement of serum lipids, plasma glucose and serum insulin concentrations. A 75 g OGTT was performed and plasma glucose and serum insulin concentrations were measured 30 and 120 min after the glucose load.

The study protocol was reviewed and approved by the ethical committee of the Saint-Louis Medical School at the Paris Diderot, University of Paris, and all participants gave written consent.

Laboratory procedures Plasma glucose, total cholesterol, HDL-cholesterol and triacylglycerol concentrations were measured with enzymatic methods. Serum insulin concentrations were measured using an immunoradiometric method (Bi-insulin IRMA; Cisbio International, Gif-sur-Yvette, France). Cross-reactivity with intact proinsulin and des31,32 proinsulin was $<1 \%$. The detection limit was $3.0 \mathrm{pmol} / 1$ and inter-assay $\mathrm{CV}$ was $<6.5 \%$.

Assessment of IR and definition of MS Insulin resistance (IR) and beta cell function was assessed by using homeostasis model assessment. Insulin resistance was calculated as HOMA-IR = fasting plasma insulin $(\mathrm{pmol} / \mathrm{l}) \times$ fasting plasma glucose $(\mathrm{mmol} / \mathrm{l}) / 135.6$. Insulin secretion was calculated as HOMA $\beta=(20 \times$ fasting plasma insulin [pmol/l] $) /($ fasting plasma glucose $(\mathrm{mmol} / \mathrm{l})-3.5)$ [23]. The disposition index (DI) was calculated as $\left(\operatorname{Ins}_{30}-\operatorname{Ins}_{0} /\right.$ Plasma glucose $_{30}$ - plasma glucose e $_{0}$ /HOMA-IR [24].

According to the National Cholesterol Education Program Adult Treatment Panel III criteria [25], individuals were considered as having MS if they had at least three of any of the following criteria: fasting blood glucose $\geq 6.1 \mathrm{mmol} / \mathrm{l}$, waist circumference $\geq 102 \mathrm{~cm}$ (men) or $88 \mathrm{~cm}$ (women), triacylglycerol $\geq 1.69 \mathrm{mmol} / 1$, HDL-cholesterol $<1.04 \mathrm{mmol} / 1$ (men) or $1.29 \mathrm{mmol} / \mathrm{l}$ (women) and $\mathrm{BP} \geq 130 / 85 \mathrm{mmHg}$ or treated hypertension.

Statistical analysis All statistical analyses were performed using SAS 9.1 software (SAS, Cary, NC, USA). Quantitative variables are expressed as means $\pm \mathrm{SD}$ and qualitative variables as numbers and percentages.

Quantitative variables were compared using a $t$ test in bivariate analyses, and a $\chi^{2}$ test was used for the comparison of class variables.

Linear regression was performed to explore the relationship between fetal environment as assessed by the two groups SGA/AGA and anthropometric data. We used a model adjusted for age, sex, BMI, socioeconomic status and family history of metabolic disorders. Residual distributions were checked for normality together with absence of residual heteroskedasticity.

To better understand the respective roles of fetal environment and gain in BMI on the development of MS, 
we designed a nested case-control study. For this analysis, we kept the 85 individuals affected by MS at the second visit and we selected from the cohort two individuals for each affected case $(n=170)$, matched for age and sex, but not affected by MS and taken as 'controls'. When more than two controls were available, the selection of two was randomly performed. ORs were estimated using conditional logistic regression adjusted for progression of BMI, fetal environment (SGA vs AGA), socioeconomic status and family history of metabolic disorders.
All tests were two-tailed and an $\alpha$ risk of $5 \%$ was considered as significant.

\section{Results}

Description of the population at both observations Table 1 summarises all clinical characteristics of the individuals at both visits. The duration of follow-up was the same in both groups: $7.5 \pm 2.6$ years for the AGA group vs $7.4 \pm 2.5$ years

Table 1 Clinical and biological characteristics of the participants at both observations

\begin{tabular}{|c|c|c|c|c|c|c|}
\hline \multirow[t]{2}{*}{ Characteristic } & \multicolumn{3}{|l|}{ First observation } & \multicolumn{3}{|c|}{ Second observation } \\
\hline & AGA & SGA & $p$ value & AGA & SGA & $p$ value \\
\hline \multicolumn{7}{|l|}{ Clinical variables } \\
\hline Total no. & 715 & 593 & & 715 & 593 & \\
\hline Age (years) & $22.1 \pm 3.8$ & $22.0 \pm 3.8$ & 0.8 & $29.6 \pm 4.1$ & $29.5 \pm 4.0$ & 0.55 \\
\hline Sex, male/female $(\%)$ & $47 / 53$ & $45 / 55$ & 0.57 & & & \\
\hline Body weight (kg) & $66.8 \pm 13.2$ & $63.2 \pm 13.8$ & 0.001 & $71.3 \pm 15.1$ & $68.2 \pm 16.3$ & 0.004 \\
\hline Body height (cm) & $171.7 \pm 8.7$ & $167.6 \pm 8.8$ & 0.001 & $171.7 \pm 8.7$ & $167.6 \pm 8.8$ & 0.001 \\
\hline BMI $\left(\mathrm{kg} / \mathrm{m}^{2}\right)$ & $22.6 \pm 3.7$ & $22.4 \pm 4.7$ & 0.46 & $24.0 \pm 4.33$ & $24.2 \pm 5.2$ & 0.64 \\
\hline Fat mass $(\%)$ & ND & ND & ND & $22.1 \pm 8.2$ & $23.1 \pm 8.9$ & 0.03 \\
\hline Waist circumference $(\mathrm{cm})$ & $78 \pm 10.7$ & $77.2 \pm 12$ & 0.23 & $83.3 \pm 12.7$ & $83.1 \pm 14.0$ & 0.79 \\
\hline Women & $74.0 \pm 10.4$ & $72.9 \pm 10.6$ & 0.15 & $77.8 \pm 11.5$ & $78.2 \pm 13.0$ & 0.72 \\
\hline Men & $82.6 \pm 9.2$ & $82.4 \pm 11.7$ & 0.88 & $89.6 \pm 10.9$ & $89.0 \pm 12.8$ & 0.84 \\
\hline Systolic BP (mmHg) & $120.7 \pm 11$ & $121.0 \pm 10$ & $0.63,0.03^{\mathrm{a}}$ & $119 \pm 12$ & $121 \pm 12$ & $0.11,0.004^{\mathrm{a}}$ \\
\hline Diastolic BP (mmHg) & $63 \pm 9.0$ & $63 \pm 9.1$ & $0.80,0.47^{\mathrm{a}}$ & $71.9 \pm 8$ & $72.5 \pm 8$ & $0.19,0.14^{\mathrm{a}}$ \\
\hline \multicolumn{7}{|l|}{ Metabolic variables } \\
\hline Total $(n)$ & 680 & 586 & & 680 & 586 & \\
\hline IGT on OGTT, $n(\%)$ & $11(1.62)$ & $23(4.06)$ & 0.008 & $30(4.41)$ & $33(5.83)$ & 0.25 \\
\hline Diabetes $(\%)$ & 1 & 1 & 0.15 & 4 & 4 & 0.89 \\
\hline Fasting plasma glucose $(\mathrm{mmol} / \mathrm{l})$ & $4.73 \pm 0.36$ & $4.75 \pm 0.41$ & $0.44,0.25^{\mathrm{a}}$ & $4.87 \pm 0.40$ & $4.90 \pm 0.43$ & $0.21,0.27^{\mathrm{a}}$ \\
\hline Fasting serum insulin (pmol/l) & $29.16 \pm 16.64$ & $32.94 \pm 23.64$ & $0.001,0.0003^{\mathrm{a}}$ & $34.86 \pm 15.96$ & $38.46 \pm 20.16$ & $0.0008,0.0002^{\mathrm{a}}$ \\
\hline 120 min plasma glucose $(\mathrm{mmol} / \mathrm{l})$ & $5.17 \pm 1.10$ & $5.33 \pm 1.15$ & $0.001,0.01^{\mathrm{a}}$ & $5.22 \pm 1.32$ & $5.39 \pm 1.37$ & $0.032,0.04^{\mathrm{a}}$ \\
\hline 120 min serum insulin $(\mathrm{pmol} / \mathrm{l})$ & $165.84 \pm 128.22$ & $198.66 \pm 209.82$ & $0.001,0.0006^{\mathrm{a}}$ & $151.5 \pm 118.98$ & $183.48 \pm 156.3$ & $0.0001,0.0001^{\mathrm{a}}$ \\
\hline HOMA-IR & $1.02 \pm 0.53$ & $1.17 \pm 0.91$ & $0.0008,0.0002^{\mathrm{a}}$ & $1.27 \pm 0.62$ & $1.41 \pm 0.79$ & $0.0007,0.004^{\mathrm{a}}$ \\
\hline НОМА- $\beta$ & $85.85 \pm 57.91$ & $101.56 \pm 143.19$ & $0.01,0.01^{\mathrm{a}}$ & $90.04 \pm 46.84$ & $99.41 \pm 73.85$ & $0.009,0.019^{\mathrm{a}}$ \\
\hline DI & $20.26 \pm 42.3$ & $18.7 \pm 69.6$ & $0.55,0.65^{\mathrm{a}}$ & $13.3 \pm 24.8$ & $12.4 \pm 25.2$ & $0.54,0.58^{\mathrm{a}}$ \\
\hline Fasting total cholesterol $(\mathrm{mmol} / \mathrm{l})$ & $4.71 \pm 0.94$ & $4.76 \pm 0.99$ & $0.30,0.17^{\mathrm{a}}$ & $4.81 \pm 0.87$ & $4.84 \pm 0.94$ & $0.65,0.51^{\mathrm{a}}$ \\
\hline Fasting HDL-cholesterol (mmol/l) & $1.43 \pm 0.34$ & $1.40 \pm 0.36$ & $0.16,0.23^{\mathrm{a}}$ & $1.36 \pm 0.31$ & $1.32 \pm 0.32$ & $0.03,0.03^{\mathrm{a}}$ \\
\hline Fasting triacylglycerol (mmol/l) & $1.01 \pm 0.50$ & $1.09 \pm 0.54$ & $0.009,0.005^{\mathrm{a}}$ & $1.04 \pm 0.77$ & $1.12 \pm 0.89$ & $0.09,0.11^{\mathrm{a}}$ \\
\hline \multicolumn{7}{|l|}{ MS } \\
\hline Total $(n)$ & 697 & 576 & & 697 & 576 & \\
\hline$n(\%)$ & $1(0.14)$ & $15(2.60)$ & $0.004,0.005^{\mathrm{a}}$ & $34(4.88)$ & $51(8.85)$ & $0.001,0.05^{\mathrm{a}}$ \\
\hline
\end{tabular}

Values are means $\pm \mathrm{SD}$

$p$ values for bivariate $t$ tests or $\chi^{2}$ tests

${ }^{a}$ The second $p$ values are further adjusted for sex, age, BMI, socioeconomic status and family history of metabolic disorders and further adjusted for height for BP

IGT, impaired glucose tolerance; ND, not determined 
for the SGA group $(p=0.49)$. Of the participants, 593 SGA and 715 AGA were studied at 22 and 29 years of age. The proportion of men and women was comparable in the two groups. Body weight and height were significantly decreased at both time-points in the SGA group, so that BMIs were similar at both observations. As previously described, percentage of body fat was significantly increased in the SGA group.

Systolic BP was significantly increased in the SGA group at both observations whereas diastolic BP was not, and the proportion of individuals receiving anti-hypertensive treatment was $<1 \%$ in both groups (seven individuals born AGA vs five born SGA).

The number of individuals with diabetes was the same in the two groups (four individuals developed the disease in each group). In the AGA group, all individuals were diagnosed on OGTT at the second visit. In the SGA group, one individual was known to have been diabetic for 3 years and was receiving glucose-lowering drugs (metformin, pioglitazone) and insulin glargine (A21Gly,B31Arg, B32Arg human insulin); he was therefore excluded from the tests. The others were diagnosed on OGTT.

For the metabolic variables, we analysed only the individuals with complete data at both observations. The prevalence of impaired glucose tolerance differed significantly between the AGA and the SGA groups at the first observation, but became similar at the second observation (Table 1). However, plasma glucose and insulin concentrations profiles during OGTT remained comparable at age 22 and 29 years. Individuals born SGA had fasting plasma glucose similar to individuals born AGA, but significantly higher plasma glucose concentrations at $120 \mathrm{~min}$ of an OGTT. Fasting plasma insulin was significantly increased in the SGA group, even after adjusting for sex, age, BMI, socioeconomic status and family history. HOMA-IR, taken as an index of IR, was significantly increased in individuals born SGA, at both visits. Insulin secretion, assessed by
HOMA- $\beta$, was found significantly elevated in the SGA group at the two time-points. Consequently, DI did not significantly differ between the two groups at the two observations.

The lipid profiles were comparable in the SGA and AGA groups at both time-points for plasma total cholesterol. The plasma HDL-cholesterol concentration became significantly decreased and plasma triacylglycerol concentration remained increased, but not significantly, in the SGA group at the second observation. In both groups, the number of individuals using lipid-lowering medication was negligible (five patients from the AGA group vs four in the SGA group).

Prevalence of MS At both observations, individuals born SGA had a significantly increased prevalence of MS. At the first observation, only one individual from the AGA group $(0.14 \%)$ and 15 individuals born SGA $(2.60 \%)$ were classified as having MS. All of these individuals had three criteria for the diagnosis of MS. After 7.5 years, 31 individuals born AGA (4.45\%) and 39 (6.77\%) individuals born SGA had three criteria of MS, and three individuals $(0.43 \%)$ born AGA compared with 12 individuals $(2.08 \%)$ born SGA described four criteria of MS.

Progression of the metabolic variables over time and effect of gain in BMI To better understand the role of gain in BMI during the follow-up, the entire cohort was divided into tertiles of gain in BMI between the two observations. The progression of IR, evaluated by change in HOMA-IR, was not significantly different across the tertiles of change in BMI in the two groups. The interaction between fetal environment (AGA/SGA) and the progression of metabolic disorders were not significant either for the progression of MS or for the progression of IR (data not shown).

To analyse further the respective roles of fetal environment and the gain in BMI during the follow-up, a nested case-control study was designed (see Statistical analysis

Table 2 Nested case-control study of participants with MS or not at the second visit

\begin{tabular}{|c|c|c|c|c|c|}
\hline \multirow[t]{2}{*}{ Co-variable } & \multirow[t]{2}{*}{ MS at second visit, $n(\%)$} & \multicolumn{2}{|l|}{ Bivariate } & \multicolumn{2}{|l|}{ Mutivariable $^{\mathrm{a}}$} \\
\hline & & OR $(95 \% \mathrm{CI})$ & $p$ value & OR $(95 \% \mathrm{CI})$ & $p$ value \\
\hline \multicolumn{6}{|l|}{ Change in BMI } \\
\hline First tertile & $5(1.2)$ & 1 & - & 1 & - \\
\hline Second tertile & $22(5.3)$ & $5.02(1.81-13.91)$ & 0.002 & $4.97(1.69-14.60)$ & 0.004 \\
\hline Third tertile & $58(12.5)$ & $10.69(4.00-28.58)$ & $<0.0001$ & $9.92(3.55-27.71)$ & $<0.0001$ \\
\hline \multicolumn{6}{|l|}{ Group } \\
\hline AGA & $34(4.8)$ & 1 & 0.01 & 1 & 0.047 \\
\hline SGA & $51(8.7)$ & $2.05(1.20-3.48)$ & & $1.93(1.01-3.68)$ & \\
\hline
\end{tabular}

${ }^{a}$ Further adjusted for group AGA/SGA, change in tertile BMI, socioeconomic status and family history of metabolic disorders 
section). Eighty-five individuals with MS were compared with 170 controls without MS matched for sex and age. In this analysis, being born SGA conferred a 1.9-fold higher risk of having MS at the second visit in comparison with AGA (OR 1.93, 95\% CI 1.01-3.68; $p=0.04$ after adjustment for change in BMI and other confounding factors; see Table 2).

\section{Discussion}

At 30 years of age, individuals born SGA remain more insulin-resistant and more frequently affected by MS than paired adults born AGA. The higher progression of BMI at the expense of a greater fat mass tends to overshadow these results in comparison with what was observed at the age of 22 years. However, the effect of being born SGA remains a significant contributor to the risk of MS, despite the progression of BMI; this effect was not observed for IR over the relative short period of time for the present follow-up.

The strength of our observations relies on the study design. This is a community-based cohort with men and women recruited from a maternity registry with no particular medical follow-up later in life for any clinical condition. The study population covers a very homogeneous population in the Alsace basin in the east of France. The high rate of participation at the second visit $(>80 \%)$, similar in the two groups, makes unlikely a strong selection bias during the follow-up. Moreover, some individuals who declined to participate could be reached by phone. The selfreported anthropometric data of these individuals do not differ from those in the active participants. This cohort is unique for other reasons as well. The two groups of individuals have been carefully selected on birth data from a maternity registry. Children grew up at a time (1970s and 1980s) when nutritional conditions were optimal and quite uniform in the small area of Haguenau. The individuals are now in their early thirties, at which age pubertal development of body composition has been completed. Additionally, the metabolic status of the individuals is not yet confounded by the development of diabetes during ageing.

MS is a concurrence of usually mild and varying degrees of disturbed glucose and insulin metabolism, overweight and abdominal fat distribution, dyslipidaemia and hypertension. A small proportion of the individuals of the cohort were affected by this syndrome (31 individuals born AGA [4.45\%] and 39 [6.77\%] individuals born SGA had three criteria of MS and three individuals [0.43\%] born AGA compared with 12 individuals [2.08\%] born SGA described four criteria of MS). Other epidemiological data have confirmed the association between components of MS and BW. But, because of the lack of an accepted definition, most of the studies differed in their definition of MS [3, 6 ,
14, 26], so that direct comparison is difficult. However, the twofold increased prevalence of MS is comparable with what is reported in the literature $[6,27]$.

The specific point of this study is that we describe the progression of MS in a cohort of young adults with respect to the in utero environment. Byberg et al. [26] reported a prospective follow-up of men investigated at 50 and 70 years of age. Unfortunately, the investigations performed at the two examinations were not similar, so that the authors could not describe the progression of the metabolic variables during the follow-up. In this study, BW was inversely related to the development of 'IR' syndrome at both ages.

It is generally accepted that the metabolic consequences of low BW are linked to the postnatal catch-up, which in turn is associated with increased adiposity in humans and animals models [28, 29]. Indeed, this phenomenon of preferential catch-up in fat is intimately associated with IR, and IR appears as an early feature in individuals born SGA, as early as 1 and 2 years of age [12]. However, we show here that this phenomenon can extend to young adulthood far beyond the phase of catch-up. Moreover, our data suggest independent effects of the intra-uterine environment and of later weight gain on the progression of MS in adults born SGA.

In conclusion, at both ages 22 and 30 years, individuals born SGA are more insulin-resistant and have a significantly higher prevalence of MS in comparison with pairs born AGA, despite a very steep progression of MS in the AGA group. This higher progression of MS in SGA individuals seems related to both an increased weight gain and to fetal programming itself.

Acknowledgements The authors are grateful to C. Trabant, M. Grasser and S. Wendling for their nursing skills and to the nursing and medical staff of the Clinical Investigation Centre 92-02 (Robert Debré Hospital, Paris), who performed medical and biological tests. This study was supported by a grant from the Agence Nationale de la Recherche (ANR-06-Physio 037), from INSERM (COSSEC 2007) and by a research grant from Pfizer France.

Duality of interest The authors declare that there is no duality of interest associated with this manuscript.

\section{References}

1. Phillips DI, Barker DJ, Hales CN, Hirst S, Osmond C (1994) Thinness at birth and insulin resistance in adult life. Diabetologia $37: 150-154$

2. Barker DJ (2004) The developmental origins of chronic adult disease. Acta Paediatr Suppl 93:26-33

3. Barker DJ, Hales CN, Fall CH, Osmond C, Phipps K, Clark PM (1993) Type 2 (non-insulin-dependent) diabetes mellitus, hyper- 
tension and hyperlipidaemia (syndrome $\mathrm{X}$ ): relation to reduced fetal growth. Diabetologia 36:62-67

4. Reinehr T, Kleber M, Toschke AM (2009) Small for gestational age status is associated with metabolic syndrome in overweight children. Eur J Endocrinol 160:579-584

5. Leon DA, Koupilova I, Lithell HO et al (1996) Failure to realise growth potential in utero and adult obesity in relation to blood pressure in 50 year old Swedish men. BMJ 312:401-406

6. Valdez R, Athens MA, Thompson GH, Bradshaw BS, Stern MP (1994) Birthweight and adult health outcomes in a biethnic population in the USA. Diabetologia 37:624-631

7. Jaquet D, Deghmoun S, Chevenne D, Collin D, Czernichow P, Levy-Marchal C (2005) Dynamic change in adiposity from fetal to postnatal life is involved in the metabolic syndrome associated with reduced fetal growth. Diabetologia 48:849-855

8. Enzi G, Zanardo V, Caretta F, Inelmen EM, Rubaltelli F (1981) Intrauterine growth and adipose tissue development. Am J Clin Nutr 34:1785-1790

9. Hediger ML, Overpeck MD, Kuczmarski RJ, McGlynn A, Maurer KR, Davis WW (1998) Muscularity and fatness of infants and young children born small- or large-for-gestational-age. Pediatrics 102:E60

10. Albertsson-Wikland K, Karlberg J (1994) Natural growth in children born small for gestational age with and without catchup growth. Acta Paediatr Suppl 399:64-70, discussion 71

11. Druet C, Ong KK (2008) Early childhood predictors of adult body composition. Best Pract Res Clin Endocrinol Metab 22:489-502

12. Ibanez L, Ong K, Dunger DB, de Zegher F (2006) Early development of adiposity and insulin resistance after catch-up weight gain in small-for-gestational-age children. J Clin Endocrinol Metab 91:2153-2158

13. Parker L, Lamont DW, Unwin N et al (2003) A lifecourse study of risk for hyperinsulinaemia, dyslipidaemia and obesity (the central metabolic syndrome) at age 49-51 years. Diabet Med 20:406-415

14. Barker DJ, Eriksson JG, Forsen T, Osmond C (2002) Fetal origins of adult disease: strength of effects and biological basis. Int $\mathrm{J}$ Epidemiol 31:1235-1239

15. Eriksson JG, Forsen T, Tuomilehto J, Jaddoe VW, Osmond C, Barker DJ (2002) Effects of size at birth and childhood growth on the insulin resistance syndrome in elderly individuals. Diabetologia $45: 342-348$

16. Bavdekar A, Yajnik CS, Fall CH et al (1999) Insulin resistance syndrome in 8-year-old Indian children: small at birth, big at 8 years, or both? Diabetes 48:2422-2429

17. Crowther NJ, Cameron N, Trusler J, Gray IP (1998) Association between poor glucose tolerance and rapid post natal weight gain in seven-year-old children. Diabetologia 41:1163-1167
18. Levitt NS, Lambert EV, Woods D, Seckl JR, Hales CN (2005) Adult BMI and fat distribution but not height amplify the effect of low birthweight on insulin resistance and increased blood pressure in 20-year-old South Africans. Diabetologia 48:1118-1125

19. Kensara OA, Wootton SA, Phillips DI, Patel M, Jackson AA, Elia M (2005) Fetal programming of body composition: relation between birth weight and body composition measured with dual-energy X-ray absorptiometry and anthropometric methods in older Englishmen. Am J Clin Nutr 82:980-987

20. Yajnik CS (2004) Early life origins of insulin resistance and type 2 diabetes in India and other Asian countries. J Nutr 134:205-210

21. Meas T, Deghmoun S, Armoogum P, Alberti C, Levy-Marchal C (2008) Consequences of being born small for gestational age on body composition: an 8-year follow-up study. J Clin Endocrinol Metab 93:3804-3809

22. de Rooij SR, Painter RC, Roseboom TJ et al (2006) Glucose tolerance at age 58 and the decline of glucose tolerance in comparison with age 50 in people prenatally exposed to the Dutch famine. Diabetologia 49:637-643

23. Matthews DR, Hosker JP, Rudenski AS, Naylor BA, Treacher DF, Turner RC (1985) Homeostasis model assessment: insulin resistance and beta-cell function from fasting plasma glucose and insulin concentrations in man. Diabetologia 28:412-419

24. Kahn SE (2003) The relative contributions of insulin resistance and beta-cell dysfunction to the pathophysiology of type 2 diabetes. Diabetologia 46:3-19

25. National Cholesterol Education Program (2001) Executive summary of the third report of The National Cholesterol Education Program (NCEP) expert panel on detection, evaluation, and treatment of high blood cholesterol in adults (Adult Treatment Panel III). JAMA 285:2486-2497

26. Byberg L, McKeigue PM, Zethelius B, Lithell HO (2000) Birth weight and the insulin resistance syndrome: association of low birth weight with truncal obesity and raised plasminogen activator inhibitor-1 but not with abdominal obesity or plasma lipid disturbances. Diabetologia 43:54-60

27. Ramadhani MK, Grobbee DE, Bots ML et al (2006) Lower birth weight predicts metabolic syndrome in young adults: the Atherosclerosis Risk in Young Adults (ARYA)-study. Atherosclerosis $184: 21-27$

28. Desai M, Crowther NJ, Lucas A, Hales CN (1996) Organselective growth in the offspring of protein-restricted mothers. $\mathrm{Br}$ J Nutr 76:591-603

29. Desai M, Gayle D, Babu J, Ross MG (2005) Programmed obesity in intrauterine growth-restricted newborns: modulation by newborn nutrition. Am J Physiol 288:R91-R96 